\section{Atomic Resolution of the Silicon (111)-(7×7) Surface by Atomic Force Microscopy}

\author{
Franz J. Giessibl
}

Achieving high resolution under ultrahigh-vacuum conditions with the force microscope can be difficult for reactive surfaces, where the interaction forces between the tip and the samples can be relatively large. A force detection scheme that makes use of a modified cantilever beam and senses the force gradient through frequency modulation is described. The reconstructed silicon (111)- $(7 \times 7)$ surface was imaged in a noncontact mode by force microscopy with atomic resolution ( 6 angstroms lateral, 0.1 angstrom vertical).

The atomic resolution of the scanning tunneling microscope (STM) allowed a longstanding question, the nature of the surface reconstruction of $\mathrm{Si}(111)-(7 \times 7)(1)$, to be resolved. Achieving similar resolution with the atomic force microscope (AFM) (2) under ultrahigh vacuum (UHV) has proven more difficult. The operation of an AFM is based on bringing a tip in close proximity to a surface and scanning while controlling the distance for a constant interaction force. The tip is usually mounted on a cantilever beam (CL). Progress in force microscopy toward true atomic resolution has been slower for two main reasons: (i) The nature of the forces between a tip and a sample is more complex than the tunneling current between a well-conducting tip and sample, and (ii) it is relatively easy to measure the tunneling currents (nanoampere range) with a good signal-to-noise $(\mathrm{S} / \mathrm{N})$ ratio, whereas measuring the forces required for atomic resolution imaging (nanonewton range) is a much more challenging problem, especially in UHV.

Initial reports of "atomic" resolution by an AFM in vacuum showed the periodic lattice of $\mathrm{NaCl}(001)$ (3), but these images did not show any singularities like defects or steps. The AFM was operating in the contact mode, where the tip and sample were in mechanical contact. This mode works well in ambient conditions because most surfaces are covered with a layer of water, hydrocarbons, or other contaminants when exposed to air. In UHV, clean surfaces tend to stick together, especially when the materials are identical. Therefore, the choice of sample and tip material is important. Ionic $\mathrm{NaCl}$ crystals are chemically inert, and close contact between tip and sample does not create chemical bonds. The interaction forces were on the order of $10 \mathrm{nN}$, about 100 times greater than what was acceptable for a single-atom tip. Many "minitips" must have been in contact with the surface, which compromises and limits the observation of steps and defects. In order to achieve

$\overline{\text { Park Scientific Instruments, } 1171 \text { Borregas Avenue, }}$ Sunnyvale, CA 94089, USA. true atomic resolution, imaging must proceed with very small interaction forces. Forces in an AFM cannot be gauged directly; they are usually determined by measuring the deflection of a spring. Absolute length measurements of objects the size of centimeters on a nanometer scale are subject to thermal drift. For that reason, it is very difficult to maintain a constant interaction force while taking an image in contact force microscopy. The weaker the spring constant, the less critical the length measurement becomes. However, the tip is subject to attractive van der Waals interactions before it makes contact with the surface. When the force gradient of the attractive interaction exceeds the spring constant of the CL, the tip snaps into contact, which can crush an initially sharp tip. This is less likely to happen for stiff CLs.

The van der Waals forces can be reduced by performing the experiments in water. Ohnesorge and Binnig (4) demonstrated true atomic resolution with an AFM by imaging steps on a calcite crystal in water. They could resolve the unit cell in both attractive and repulsive modes. The homemade instrument used CLs with very sharp tips (5). The repulsive forces between tip and sample were $<0.1 \mathrm{nN}$. In UHV, the only way to reduce the van der Waals forces is to use very sharp tips. The problem of maintaining a small repulsive force for the time required to take an image is still present. One solution to that problem is to operate the AFM at low temperature (6) to minimize thermal drifts. A different approach is to use an ac method. These problems and their possible solutions were already pointed out by Binnig et al. (2). Atomic resolution of the $\mathrm{Si}(111)-(7 \times 7)$ reconstruction by AFM is challenging because this surface is reactive. Meyer and co-workers (7) studied the $\mathrm{Si}(111)-(7 \times 7)$ reconstruction in UHV by contact force microscopy and observed adhesive forces of up to $10^{3} \mathrm{nN}$ between a $\mathrm{Si}$ tip and the Si(111) surface. By coating the tip with polytrifluoroethylene (Teflon), they could reduce the sticking forces to $10 \mathrm{nN}$. The periodicity of the unit cell could be resolved by using coated tips, but the images show friction effects. Thus, mechanical contact between tip and sample must be avoided for the imaging of a reactive surface. A noncontact method has allowed imaging of the rows of the unit cells of $\mathrm{Si}(111)-(7 \times 7)(8)$.

The data presented in this report were taken with the AutoProbe VP (9), a combined STM and AFM for UHV, in standard configuration. The experimental setup is described in more detail in (10). The instrument uses a force detection scheme that simplifies the operation in UHV considerably. The CLs are etched out of singlecrystalline $\mathrm{Si}$, have a conductive channel (doped $\mathrm{Si}$ ) on one side, which changes its resistance when strained, and also incorporate a very sharp integrated tip (11). This so-called Piezolever (PL) is part of a Wheatstone bridge. We have chosen the frequency modulation $(\mathrm{fm})$ noncontact method introduced by Albrecht et al. (12). In that method, the spring that carries the AFM tip is subject to positive feedback and thus oscillates at its eigenfrequency (13). The positive feedback mechanism maintains a constant amplitude. The data shown here have been taken with PLs with an eigenfrequency of $\sim 120 \mathrm{kHz}$ (14). The eigenfrequency $\nu_{0}$ of the PL is given by

$$
\nu_{0}=2 \pi \sqrt{\frac{k_{\mathrm{PL}}}{m}}
$$

where $k_{\mathrm{PL}}$ is the spring constant of the lever and $m$ is the reduced mass. When the sample approaches the tip, the force gradient $\Delta k=\partial F_{\text {tip-sample }} / \partial z$ of the tip-sample interaction (resulting from van der Waals and electrostatic forces) alters the effective force constant, and the frequency changes according to

$$
\frac{\Delta v}{v_{0}}=\frac{\Delta k}{2 k_{\mathrm{PL}}}
$$

when $\Delta k / k_{\mathrm{PL}} \ll 1$. The tip-sample interaction is in general attractive before the tip reaches contact; therefore, the frequency decreases as the sample approaches the tip. The fm noncontact AFM allows the imaging of a surface at a constant frequency shift and thus creates a map of a constant average force gradient.

There are three externally adjustable parameters that affect the imaging process: the oscillation amplitude, the set point of the frequency shift, and the bias between tip and sample. To achieve optimal resolution, the bias is set to zero. The amplitude and frequency shift affect each other because the gradient of the tip-sample interaction varies with distance. For a set minimum distance between tip and sample, the frequency shift decreases as the amplitude increases. The best combination between $\Delta v$ and the oscillation amplitude is found em- 
pirically. An amplitude of $\sim 200 \AA$ and a relative frequency shift of $-0.01 \%$ turned out to be a good starting point. The noise in the surface normal $(z)$ position is minimized by varying both the amplitude and the frequency shift.

The fm noncontact method has three advantages over the contact AFM: (i) The eigenfrequency of the $\mathrm{PL}$ is much higher than the $1 / f$ corner frequency $(11)$, the $1 / f$ noise prevalent in contact force microscopy is outside of the bandwidth of the fm detector, and the noise in the fm detector is thermally limited (15). (ii) The tip does not touch the sample during imaging (no danger of chemical bonding between tip and sample). (iii) The fm method is sensitive to the force gradient rather than the force. Sensing the force gradient rather than the force increases the sensitivity to forces that are spatially dependent on the atomic scale. These three benefits are important for achieving ultimate resolution at small interaction forces (8).

An image of $\mathrm{Si}(111)-(7 \times 7)(16)$ taken with an STM with positive sample bias is shown in Fig. 1. The short and the long diagonals of the unit cell are depicted in the image. The diagonals end in the so-called cornerholes. The protrusions in the image are the 12 adatoms per unit cell according to the dimer-adatom-stacking fault (DAS) model (17). According to that model, out of the 49 surface atoms in one unit cell, 12 of them (the so-called adatoms) sit in the top layer. The four valence electrons in $\mathrm{Si}$ form $s p^{3}$ hybridized orbitals in tetrahedral configuration, so the fourth orbital does not have a binding partner and is perpendicular to the surface (dangling bond). The depth of each cornerhole is $\sim 2 \AA$.

The AFM noncontact image of Si(111)$(7 \times 7)$ in Fig. 2 represents the data with the highest $\mathrm{S} / \mathrm{N}$ ratio that was obtained on this sample. The image was taken in the topographic mode; that is, the distance between the sample and the median position of the PL was adjusted during the scan for a constant frequency shift. The lower section of the image shows $7 \times 7$ unit cells but a typical multitip image. In the upper section of the image, the tip was monatomic for about the width of a unit cell. This phenomenon is also commonly observed with an STM: The tip suddenly switched from a multitip (several atoms on the tip were contributing significantly to the image) to a monatomic tip. After extended scanning over the surface, the resolution deteriorated. The tip probably picked up contaminants. So far, I have reproduced atomic resolution on this surface with the AFM three times in addition to Fig. 2. Progress in obtaining larger areas with atomic resolution can be expected in analogy to the development of atomic resolution imaging of $\mathrm{Si}(111)-(7 \times 7)$ by
STMs: The first atomic resolution image (1), and with the evolution of recipes for preparing tips and samples, larger areas will be imaged with atomic resolution by the AFM.

The AFM image shows 12 protrusions per unit cell. I believe that these protrusions are the dangling bonds of the adatom layer in the DAS model and suggest that their high polarizability is responsible for the compare well to STM images taken with positive sample bias. The depth of the cornerholes is $\sim 1 \AA$ as opposed to the value of taken by an STM was $\sim 3$ unit cells in size contrast in the image. The AFM images

$\sim 2 \AA$ obtained with an STM. This difference may result from the lesser dependence of the error signal with distance compared with that for the STM; as the foremost tip atom "dives" into the cornerhole, other tip atoms interact with the adatoms and cause a smaller apparent corrugation.

For an understanding of the imaging process in any scanning probe technique, it is crucial to analyze the variation of the signal that is used to obtain the image as a function of the distance of the probe to the surface. Figure 3 shows the natural logarithm of the relative frequency change versus distance. These data were obtained by

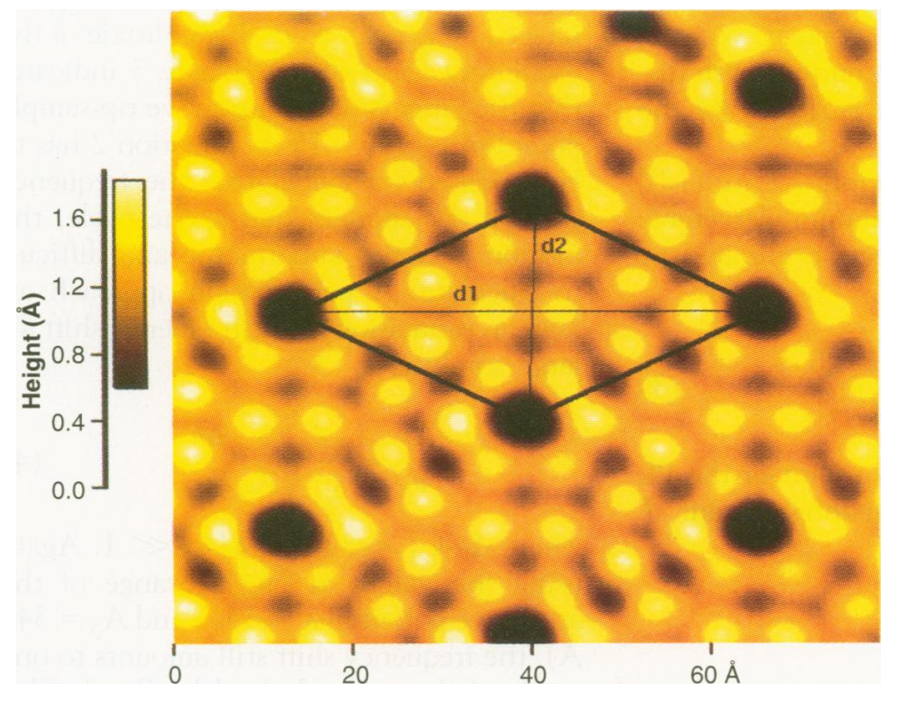

Fig. 1. An STM image of Si(111)- $(7 \times 7)$ taken at a sample bias of $+1.96 \mathrm{~V}$ and a current of $400 \mathrm{pA}$. Image size is $78 \AA$ by 78 $\AA$. The unit cell is indicated by the black diamond. The lengths of the diagonals are $d_{1}=46.6$ $\AA$ and $d_{2}=26.9 \AA$. The image shows the 12 adatoms per unit cell. The black spots, called cornerholes, each have a depth of $\sim 2 \AA$.

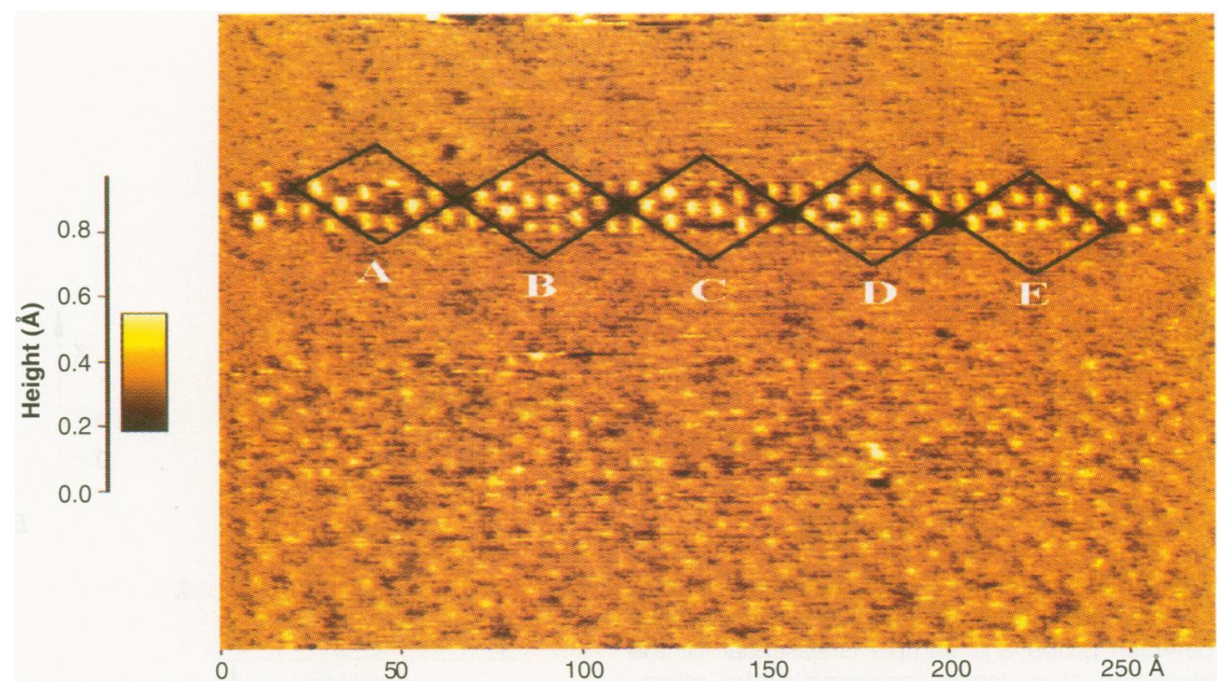

Fig. 2. Noncontact image of $\mathrm{Si}(111)-(7 \times 7)$ (unfiltered data, corrected for thermal drift). Image size is 270 $\AA$ by $195 \AA$. The sample bias was adjusted to match the tip potential and thus minimize the electrostatic interaction (8). Imaging parameters: $A_{0}=340 \AA, v_{0}=114,224 \mathrm{~Hz}, \Delta v=-70 \mathrm{~Hz}$, and the force constant of the $P L$ is $17 \mathrm{~N} / \mathrm{m}$. The image shows a phenomenon that is well known from STM imaging: The tip suddenly switches from a multitip to a single tip. The fast scan direction is from left to right $(3.2 \mathrm{~Hz})$, the slow scan from bottom to top. The lower part of the image shows a typical multitip image of $\mathrm{Si}(111)-(7 \times 7)$, then the tip quality deteriorated, and suddenly a monotip created a crisp image of the adatom structure for a height of about one unit cell. Six cornerholes, embracing five unit cells, are clearly visible. The five unit cells also show the adatom structure similar to the STM image in Fig. 1. Unit cell C indicates all 12 adatoms. Unit cells $D$ and $E$ show the two adatoms that are not visible in $A$ and $B$. Unit cell $A$ shows an atomic defect. The right of the two central adatoms is misplaced. 
presetting the frequency shift and measuring the position of the sample in the $z$ (vertical) direction. The frequency shift increases sharply with decreasing distance until the feedback becomes unstable at distance $D_{0}$. It is not possible to determine the absolute value of $D_{0}$, but because we could use a PL with a similar spring constant and tip shape for tunneling (10), I assume that $D_{0}$ is the distance where the PL starts to snap into the surface. Because I could obtain STM images and the distances for tunneling are on the order of $5 \AA$, I assume that $D_{0}$ is less than $5 \AA$ because in the tunneling experiments, the electrostatic interaction (which was zero in the AFM experiment because of zero bias) was added to the attractive interaction. For comparison, the variation of the tunneling current with distance for a metal sample is shown by the dashed line in Fig. 3.

Albrecht et al. [equation 19 in (12)] have calculated the minimum detectable force gradient in $\mathrm{fm}$ detection (limited by thermal detector noise)

$$
\Delta k_{\text {min }}=\sqrt{\frac{4 k_{\mathrm{PL}} k_{\mathrm{B}} T \mathrm{~B}}{\pi v_{0} \mathrm{QA}_{0}^{2}}}
$$

where $k_{\mathrm{PL}}$ is the spring constant of the PL $(17 \mathrm{~N} / \mathrm{m}), k_{\mathrm{B}}$ is the Boltzmann constant, $T$

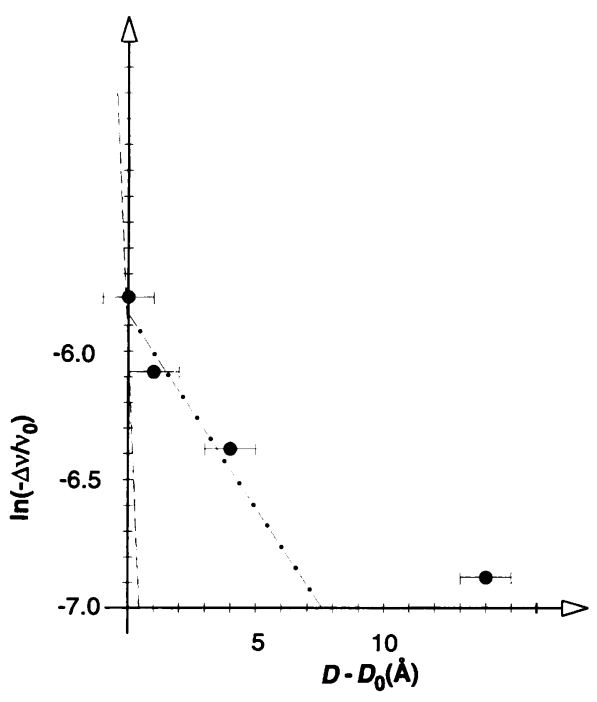

Fig. 3. The dependence of the signal that is used to derive the image with respect to the distance between probe and sample is essential for the resolution of a general scanning probe microscope. This graph shows the natural logarithm of the negative relative frequency shift of the $P L$ as a function of distance. At $D=D_{0}$, the feedback becomes unstable. As a comparison, the dashed line marks the dependence of the tunneling current with distance in the case of the STM for a metallic surface. The dash-dotted line is used for approximating the total force acting between tip and sample. With the use of that relation, the total force acting on the $\mathrm{PL}$ is $-0.14 \mathrm{nN}$ (attractive) when the PL tip is closest to the sample. is temperature (in kelvin) $(300 \mathrm{~K}), B$ is the bandwidth of the detection circuit $(1 \mathrm{kHz})$, $\nu_{0}$ is the eigenfrequency of the PL (114 $\mathrm{kHz}), \mathrm{Q}$ is the quality factor of the PL $[28,000(10)]$, and $A_{0}$ is the oscillation amplitude of the PL ( $340 \AA$ ). Equation 3 yields $\Delta k_{\min }=4.9 \times 10^{-6} \mathrm{~N} / \mathrm{m}$ (using the values given above in parentheses, which were used when Fig. 2 was obtained).

According to Eq. 3, the minimal detectable force gradient is proportional to the inverse of the oscillation amplitude of the PL. However, a large amplitude implies that the tip of the PL is only affected by the sample during a part of its oscillation cycle. Figure 4A visualizes the geometric relations between tip and sample when the sample is imaged. The peak-to-peak amplitude of the oscillating PL is $680 \AA$. Figure 3 indicates that the range $\lambda$ of the attractive tip-sample potential is only $\sim 15 \AA$. Equation 2 has to be modified for calculating the frequency shift. If $\Delta k$ changes during the cycle, the motion becomes anharmonic and difficult to calculate. A perturbation approach allarge amplitudes

$$
\frac{\Delta v}{\nu_{0}} \approx \frac{\sqrt{2}}{\pi} \sqrt{\frac{\lambda}{\mathrm{A}_{0}}} \frac{\Delta k}{2 k_{\mathrm{PL}}}
$$

where $\Delta k / k_{\mathrm{PL}} \ll 1$ and $\lambda / A_{0} \ll 1$. Again using the values of Fig. 2 (range of the attractive potential $\lambda=15 \AA$ and $A_{0}=340$ $\AA$ ), the frequency shift still amounts to one tenth of the value derived by Eq. 2 . This result may seem surprising, but the velocity lows an estimation of the frequency shift for

of the tip is zero at its turning points, and therefore, the tip spends a relatively long time close to the sample, even though the mean distance is large. Clamping a ruler at the edge of a desk and exciting it to oscillate causes the same phenomenon: The ruler seems to split into two pieces at the turnaround points. Combining the findings in Eqs. 3 and 4 would still favor infinitely large amplitudes $A_{0}$ for the detection of the weakest possible force gradients. However, other sources of noise (like the drift of $\nu_{0}$ with temperature) become noticeable as $A_{0}$ is set to larger values.

A detailed view of the tip and sample when the PL is at its close turnaround point is shown in Fig. 4B. The average tip radius is based on the estimate in (10); we were using similar PL tips. It has been shown that for an exponentially dependent tip-sample interaction, a tip composed of a single atom at the end of tip with a radius of $300 \AA$ will still produce atomic resolution (18).

The maximum force that can be exerted from a tip atom to a sample atom without breaking the bonds between the surface atom and the underlying layer is an important parameter. A simple model may give some insight into that complicated matter. Silicon has a cohesive energy of $7.4 \times$ $10^{-19} \mathrm{~J}[4.63 \mathrm{eV}(19)]$ per atom. Thus, each bond has a binding energy of $-3.7 \times 10^{-19}$ $\mathrm{J}$. Assuming a linear potential with a range of $0.37 \AA$, the corresponding force is $1 \mathrm{nN}$ (20). The dash-dotted line in Fig. 3 and Eq. 4 provide an estimate for the gradient of the tip-sample interaction. Integrating that gra-

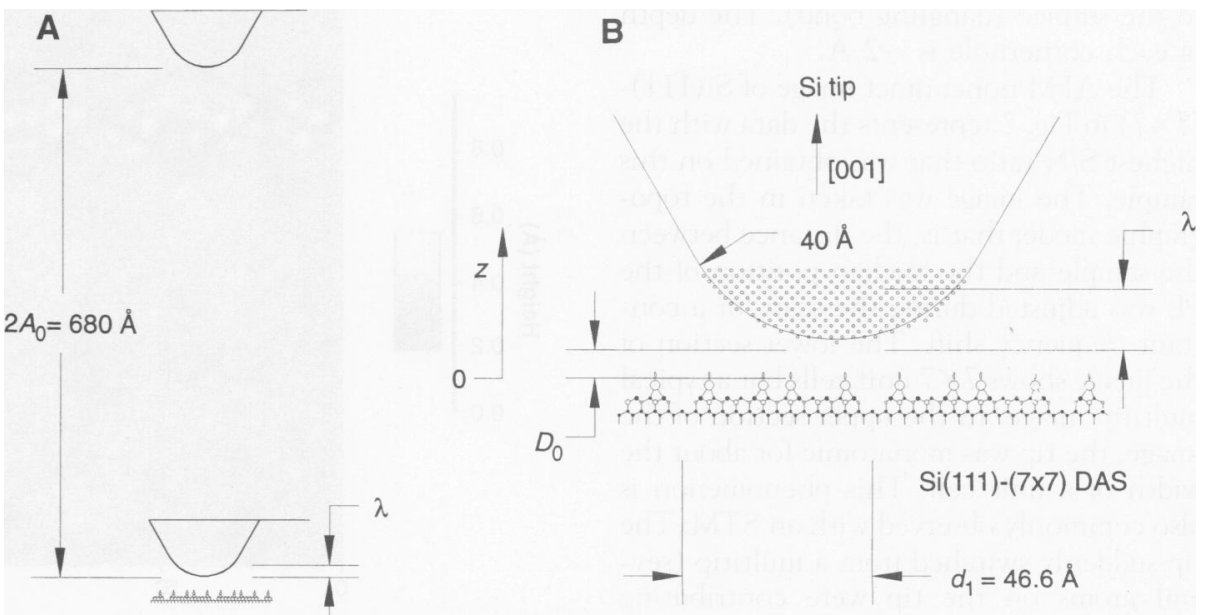

Fig. 4. Schematic of the geometric relations during imaging. (A) The PL is shown at its upper and lower turning points. The mean position of the tip is much further away from the surface than the range of the potential. Equation 2 is no longer valid for determining the frequency shift and must be replaced by a more sophisticated model. This new motion is very complex, and a simple perturbation approach (21) may serve as an estimate. (B) Schematic of the PL tip when it reaches its closest point to the sample $z=D_{0}$. The atomic positions of the surface atoms of the Si $(111)-(7 \times 7)$ reconstruction according to the DAS model are shown. The dangling bonds of the adatoms are indicated by the ellipses. The PL tip is oriented in [001] direction. The tip radius of $40 \AA$ is based on the estimate in (10). The natural cleavage planes of $\mathrm{Si}$ are $\{111\}$ planes; it could be speculated that the very end of the tip is therefore bounded by a $(11 \overline{1}), \mathrm{a}$ $(1 \overline{11})$, and $\mathrm{a}(\overline{1} 1 \overline{1})$ plane. According to (18), atomic resolution could be achieved with much larger tip radii. 
dient from $z=D_{0}$ to $z=\infty$ yields $F_{\text {tip-sample }}$ $=-0.14 \mathrm{nN}$ (attractive) as the force acting between tip and sample when the PL is closest to the surface.

\section{REFERENCES AND NOTES}

1. G. Binnig, H. Rohrer, Ch. Gerber, E. Weibel, Phys. Rev. Lett. 50, 120 (1983)

2. G. Binnig, C. F. Quate, Ch. Gerber, ibid. 56, 930 (1986).

3. G. Meyer and N. M. Amer, Appl. Phys. Lett. 56, 2100 (1990).

4. F. Ohnesorge and G. Binnig, Science 260, 1451 (1993).

5. These tips are called Ultralevers (Park Scientific Instruments, Sunnyvale, CA)

6. F. J. Giessibl and G. Binnig, Ultramicroscopy 42, 281 (1992)

7. L. Howald, R. Luethi, E. Meyer, P. Guethner, H.-J Guentherodt, Z. Phys. B 93, 267 (1994).

8. F. J. Giessibl, Jpn. J. Appl. Phys. 33, 3726 (1994)

9. AutoProbe VP 900 (Park Scientific Instruments).

10. F. J. Giessibl and B. M. Trafas, Rev. Sci. Instrum. 65, 1923 (1994)

11. M. Tortonese, R. C. Barrett, C. F. Quate, Appl. Phys Lett. 62, 834 (1993).

12. T. R. Albrecht, P. Gruetter, D. Horne, D. Rugar, J. Appl. Phys. 69, 668 (1991)

13. The forces acting between tip and sample during STM operation have been measured by mounting a sample on a $\mathrm{CL}$ and monitoring the variation of the oscillation frequency of the sample-CL assembly by $\mathrm{fm}$ detection of the tunneling current [U. Dürig, $\mathrm{O}$. Züger, D. W. Pohl, Phys. Rev. Lett. 65, 349 (1990); U. Dürig and O. Züger, Phys. Rev. B 50, 5008 (1994); and references therein].

14. VPPL4ONO (Park Scientific Instruments).

15. The dominant low-frequency noise component in $\mathrm{fm}$ detection is the variation of the eigenfrequency of the $\mathrm{PL}$ with temperature. This frequency shift is very small for appropriate oscillation amplitudes (10) compared with the frequency shift resulting from the tip-sample interaction and can be neglected.

16. The sample was prepared by cleaning a Si(111)oriented wafer in acetone and alcohol in an ultrasonic bath for $5 \mathrm{~min}$, transferring it into the vacuum chamber, and then heating it to $1170^{\circ} \mathrm{C}$ by electron beam heating. The pressure during heating increased to $1.3 \times 10^{-9} \mathrm{mbar}$. The base pressure of the vacuum system is $5 \times 10^{-11} \mathrm{mbar}$.

17. K. Takayanagi, Y. Tanishiro, M. Takahashi, S. Takahashi, J. Vac. Sci. Technol. A 3, 1502 (1985).

18. F. J. Giessibl, Phys. Rev. B 45, 13815 (1992)

19. C. Kittel, Introduction to Solid State Physics Wiley, New York, 1986), p. 55.

20. This is a very conservative estimate, because $\mathrm{Si}$ is a very brittle material, and the actual range of the attractive interatomic potential will be much shorter. For the purpose of this analysis, this estimate is sufficient because the uncertainty in the tip-sample interaction is much larger.

21. Assuming a tip-sample potential with a constant force gradient $\Delta k$, a range $\lambda$, and a tip oscillating with an elongation according to $z(t)=D_{0}+A_{0}$ $A_{0} \cos \left(2 \pi v_{0} t\right)$, the effective force constant $k=k_{\mathrm{PL}}$ $+\Delta k$ for $-t^{\star}<t \leq t^{\star}$ and $k=k_{\mathrm{P}}$ for $t^{\star}<t \leq T-$ $t^{*}$, with $t^{*}=[T /(2 \pi)] \arccos \left(1-\lambda / A_{0}\right)$ and $T=1 / \nu_{0}$ Accordingly, the resulting frequency shift will be smaller than in Eq. 2 for large oscillation amplitudes, namely

$$
\frac{\Delta v}{\nu_{0}} \approx \frac{1}{\pi} \arccos \left(1-\frac{\lambda}{A_{0}}\right) \frac{\Delta k}{2 k_{\mathrm{PL}}}
$$

where $\Delta k / k_{\mathrm{PL}} \ll 1$ and $\nu A_{0} \ll 1$. This can be further simplified [with use of $\cos x \approx 1-\left(x^{2} / 2\right)$ for $x$ $<1$ 1] to

$$
\frac{\Delta v}{\nu_{0}} \approx \frac{\sqrt{2}}{\pi} \sqrt{\frac{\lambda}{A_{0}}} \frac{\Delta k}{2 k_{\mathrm{PL}}}
$$

22. I thank C. F. Quate for his continuous support, B. M. Trafas for sharing his experience of imaging $\mathrm{Si}(111)$ $(7 \times 7)$ using the STM with me, M. D. Kirk for technical discussions and bringing his enthusiasm to this project, S. Yoshikawa for help with sample preparation, J. Nogami for useful comments, and S. Presley for his technical support. M. Tortonese supplied me with PLs and an understanding of how to use them, and T. R. Albrecht shared his knowledge of fm detection with me.

30 August 1994; accepted 31 October 1994

\section{Atomic-Scale Images of the Growth Surface of $\mathrm{Ca}_{1-x} \mathrm{Sr}_{x} \mathrm{CuO}_{2}$ Thin Films

\author{
Kazumasa Koguchi, Takuya Matsumoto, Tomoji Kawai
}

The surface microstructure of $c$-axis $(\mathrm{Ca}, \mathrm{Sr}) \mathrm{CuO}_{2}$ thin films, grown by laser molecular beam epitaxy on $\mathrm{SrTiO}_{3}(001)$ substrates, was studied by ultrahigh-vacuum scanning tunneling microscopy (STM). Images were obtained for codeposited $\mathrm{Ca}_{1-x} \mathrm{Sr}_{x} \mathrm{CuO}_{2}$ thin films, which show a layered-type growth mode. The surfaces consist of atomically flat terraces separated by steps that are one unit cell high. A pronounced dependence of the growth mechanism on the $\mathrm{Sr} / \mathrm{Ca}$ ratio of the films was observed. Atomic resolution STM images of the $\mathrm{CuO}_{2}$ sheets in the ab plane show a square lattice with an in-plane spacing of 4 angstroms; the lattice contains different concentrations of point defects, depending on the polarity of the sample-tip bias.

Since the parent compound of the cuprate superconductors, $(\mathrm{Ca}, \mathrm{Sr}) \mathrm{CuO}_{2}$, was first synthesized by Siegrist et al. (1), this material has been studied intensively. The principal reason for this interest is that this

Institute of Scientific and Industrial Research, Osaka University, Mihogaoka, Ibaraki, Osaka 567, Japan.

*To whom correspondence should be addressed. compound has the simplest oxygen defect type perovskite structure comprising the $\mathrm{CuO}_{2}$ sheets that are considered essential for high- $T_{c}$ superconductivity $\left(T_{c}\right.$ is the superconducting transition temperature). STM has been shown to be a powerful technique for imaging high- $T_{\mathrm{c}}$ cuprates on an atomic scale (2-12). Because of the simple structure of the parent compound,
STM offers the opportunity to directly probe the $\mathrm{CuO}_{2}$ sheets without the interference of intermediate oxide layers. For the purpose of the STM studies, thin film specimens are especially attractive because they provide a well-defined surface that can be preserved from vacuum-type growth conditions (13). It is also of interest to study the layer-by-layer growth mechanism as reflected in systematic changes in the surface structure.

In this report, we describe thin films of $(\mathrm{Ca}, \mathrm{Sr}) \mathrm{CuO}_{2}$ deposited on $\mathrm{SrTiO}_{3}(001)$ substrates and studied by ultrahigh-vacuum (UHV) STM. We used a combined system for laser molecular beam epitaxy (laser MBE) with reflection high-energy electron diffraction (RHEED) and UHV STM. Film surfaces of codeposited $\mathrm{Ca}_{1-x} \mathrm{Sr}_{x} \mathrm{CuO}_{2}$ films with different $\mathrm{Sr} / \mathrm{Ca}$ ratios were prepared, as well as surfaces containing an extra monolayer of $\mathrm{CuO}$. The STM images of $c$-axis-oriented codeposited $(\mathrm{Ca}, \mathrm{Sr}) \mathrm{CuO}_{2}$ films show atomically flat and well-defined terraces. The images provide information about the atomic layer epitaxy of $(\mathrm{Ca}, \mathrm{Sr}) \mathrm{CuO}_{2}$ films. These atomic-scale images show square lattices with periods that correspond to the $a$-axis lattice constant.

The $(\mathrm{Ca}, \mathrm{Sr}) \mathrm{CuO}_{2}$ thin films were prepared by laser MBE (14) with an ArF excimer laser operating at $193 \mathrm{~nm}$ and a repetition rate of 2 to $5 \mathrm{~Hz}$. Targets for the ablation were sintered disks of $\mathrm{Ca}_{1-x} \mathrm{Sr}_{x} \mathrm{CuO}_{y}(x=0.30,0.55,0.70)$, $\mathrm{CuO}$, and metallic $\mathrm{Sr}$. The ablated species were deposited on $0.01 \%$ Nb-doped Sr$\mathrm{TiO}_{3}(001)$ single crystal substrates heated to $500^{\circ} \mathrm{C}$. During deposition, $\mathrm{NO}_{2}$ gas was directed onto the substrate as an oxidizing agent for the growth of the parent compound. The background pressure during growth was $1.0 \times 10^{-5}$ torr. Before the deposition, the substrate was heated to $600^{\circ} \mathrm{C}$ under $\mathrm{NO}_{2}$ gas flow at $1.0 \times 10^{-5}$ torr for $30 \mathrm{~min}$. Then the substrate temperature was reduced to $500^{\circ} \mathrm{C}$, and a monolayer of $\mathrm{SrO}$ was deposited to stabilize the initial stages of growth $(15,16)$. The deposition of a single monolayer of $\mathrm{SrO}$ was inferred from the observation of a single RHEED intensity oscillation. During and after the deposition of the film, we repeatedly annealed the sample by interrupting the deposition for about 10 to 20 min to enhance surface migration. After the deposition of several layers of $(\mathrm{Ca}, \mathrm{Sr})$ $\mathrm{CuO}_{2}$, the sample was cooled in $\mathrm{NO}_{2}$ gas flow. Then gas flow was stopped, and the sample was transferred into the UHV STM chamber with a base pressure of less than $10^{-10}$ torr. To obtain information about the surface microstructure during atomic layer-by-layer growth, in some experiments we deposited a monolayer of 


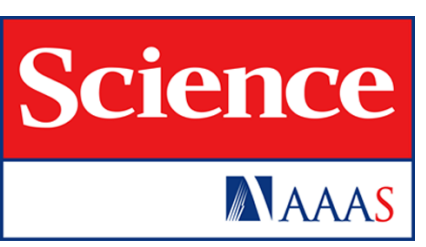

Atomic Resolution of the Silicon (111)-(7x7) Surface by Atomic Force Microscopy

Franz J. Giessibl (January 6, 1995)

Science 267 (5194), 68-71. [doi: 10.1126/science.267.5194.68]

Editor's Summary

This copy is for your personal, non-commercial use only.

Article Tools Visit the online version of this article to access the personalization and article tools:

http://science.sciencemag.org/content/267/5194/68

Permissions Obtain information about reproducing this article: http://www.sciencemag.org/about/permissions.dtl

Science (print ISSN 0036-8075; online ISSN 1095-9203) is published weekly, except the last week in December, by the American Association for the Advancement of Science, 1200 New York Avenue NW, Washington, DC 20005. Copyright 2016 by the American Association for the Advancement of Science; all rights reserved. The title Science is a registered trademark of AAAS. 